\title{
Reprint
}

\section{Cooperative Bit-loading and Fairness Bandwidth Allocation in ADSL Systems}

\author{
N. Papandreou and T. Antonakopoulos
}

\section{IEEE International Symposium on Circuits and Systems, ISCAS-2003}

\author{
BANGKOK, THAILAND, MAY 2003
}

\begin{abstract}
Copyright Notice: This material is presented to ensure timely dissemination of scholarly and technical work. Copyright and all rights therein are retained by authors or by other copyright holders. All persons copying this information are expected to adhere to the terms and constraints invoked by each author's copyright. In most cases, these works may not be reposted or mass reproduced without the explicit permission of the copyright holder.
\end{abstract}




\title{
COOPERATIVE BIT-LOADING AND FAIRNESS BANDWIDTH ALLOCATION IN ADSL SYSTEMS
}

\author{
Nikos Papandreou and Theodore Antonakopoulos \\ University of Patras, Department of Electrical Engineering and Computers Technology, \\ 26500 Rio, Patras, Greece \\ Computers Technology Institute - CTI, Riga Feraiou 61, 26100 Patras, Greece
}

\begin{abstract}
This paper examines the performance of bundled ADSL systems and proposes an extension to their bit-loading mechanism that results to fair allocation of the available bandwidth. The "ratemaximization" bit-loading method provides less average data rate compared to the optimum "target-rate" strategy, which depends on the number of active lines in the system and the length of the DSL loop. Under a non-cooperative bit-loading approach, the total data rate is not distributed uniformly among the different active lines. The individual data rate depends on the activation time of each modem relative to the total number of lines in the system. The proposed bit-loading mechanism is applied to the downstream ADSL traffic, is executed by a management unit of the central office, uses the results of the single-user bit-loading mechanism and leads to fair data rate distribution.
\end{abstract}

\section{INTRODUCTION}

In DSL systems up to 50 subscriber lines comprise a binder. The electromagnetic radiation generated in each twisted pair line induces crosstalk to the other lines in the binder [1]. This interference is one of the largest noise impairments that reduce the performance of the services sharing the same bundle. As the population of DSL services increases in the loop-plant, the need for controlling the total interference in a bundled system becomes a critical issue in order to avoid unacceptable performance degradation [2]. Crosstalk is characterized as near-end crosstalk (NEXT) generated by transmitters located on the receiver's side and far-end crosstalk (FEXT) generated by transmitters on the opposite side of the receiver. Due to channel attenuation, NEXT is in general larger than FEXT and in order to decrease its effect, DSL systems use frequency-division duplexing, so that the two directions of transmission occupy different frequency bands.

FEXT control imposes a maximum level on the transmit power of each technology in the bundle. The power spectral density (PSD) mask bounds the maximum data rate a modem can get. On the other hand, the demand for higher data rates forces the modems to claim the maximum possible rate during initialization under a "rate-maximization" strategy. Such a strategy may use all available power budget in order to achieve the maximum possible data rate [3], [4].

Early DSL systems such as asymmetric digital subscriber lines (ADSL) and high-speed digital subscriber lines (HDSL) designed as single-user systems in the sense that each modem in a bundle claims for its best performance, regardless of the other modems decisions. Recently the development of methods for coordination among the DSL modems in order to improve the system performance has become an appealing challenge [5].

In this paper we study the downstream performance of a bundled system of ADSL modems [6], which are initialized sequentially. As it is explained, the "rate-maximization" strategy does not lead to maximization of the average line rate. Other "target-rate" strategies appear to increase slightly the total binder data rate. However non-uniform distribution of the achievable data rate among the different users is noticed. This "unfairness" depends strongly on the activation time of each line relative to the total number of connections in the bundle. In this paper we show that there is a specific target-rate that maximizes the achievable total data rate and we propose an additional bit-loading mechanism that results to fair allocation of the available total data rate.

The rest of the paper proceeds as follows. Section 2 gives a description of the ADSL transmission scheme and the single-user bit-loading mechanism under the "rate-maximization" or a "target-rate" strategy. Section 3 determines the different regions of operation regarding the rate performance, discusses how the number of lines in the binder and their length affect the maximum achievable data rate, and presents the unfairness of the various "target-rate" strategies. Finally, Section 4 presents the proposed extension of the bit-loading mechanism and gives simulation results that prove its effectiveness on achieving uniform data rate distribution.

\section{THE ADSL TRANSMISSION SCHEME}

The ADSL technology uses the Discrete Multitone (DMT) transmission scheme that decomposes the channel spectrum into a set of $N=256$ independent narrowband subchannels [7]. Over a baud interval of $T$ secs (DMT block length), a sequence of $N$ complex QAM sub-symbols is assigned to these subchannels. The size of each QAM constellation depends on the channel-tonoise $(C N R)$ ratio of each subchannel and is determined during system initialization. The relationship between the number of bits and the power necessary to transmit these bits in a subchannel is given by:

$$
b_{k}=\log _{2}\left(1+\frac{P_{k} C N R_{k}}{\Gamma}\right)
$$

where $b_{k}$ is the number of bits in subchannel $k, P_{k}$ is the corresponding power, $C N R_{k}$ is the channel attenuation to noise ratio and $\Gamma$ is the so called $S N R$ gap that measures how far is the system performance from achieving its capacity. For $B E R=10^{-7}$, the $S N R$ gap is given by: 


$$
\Gamma=9.8-\gamma_{c}+\gamma_{m}
$$

The first term is related to the target symbol error rate, the term $\gamma_{c}$ depends on the applied coding scheme (e.g. Reed-Solomon) and the term $\gamma_{m}$ is the system margin, defined as the amount of noise that the system can tolerate, while still operating under the target symbol error rate. The total data rate is therefore:

$$
R=\frac{1}{T} \sum_{k=1}^{N} b_{k}=\frac{1}{T} \sum_{k=1}^{N} \log _{2}\left(1+\frac{P_{k} C N R_{k}}{\Gamma}\right)
$$

Using (1), the power $\Delta P_{k}^{A}$ needed to transmit one more bit in subchannel $k$, that already contains $b_{k}$ bits and the power $\Delta P_{k}^{R}$ saved by removing one bit are respectively:

$$
\Delta P_{k}^{A}=2^{b_{k}} \frac{\Gamma}{C N R_{k}} \text { and } \Delta P_{k}^{R}=2^{b_{k}-1} \frac{\Gamma}{C N R_{k}}
$$

\subsection{The bit-loading algorithm}

The bit-loading algorithm [4] assigns to each subchannel the lesser of the following two limits: the number of bits that can be achieved by transmitting the maximum allowable power in each subchannel as calculated by (1), and the maximum allowable number of bits as determined by the ADSL specs. After suitable power scaling to ensure integer number of bits per sub-symbol, the total power over all subchannels is calculated. If the total power is less than the power budget, the algorithm has achieved "rate-maximization" bit allocation. If the total power is greater than the power budget or a different "target-rate" is desired, the algorithm removes as many bits as necessary to meet the power or the rate constraint. The bit-removal process is performed one bit at a time, so that the most power expensive bits among all subchannels are removed.

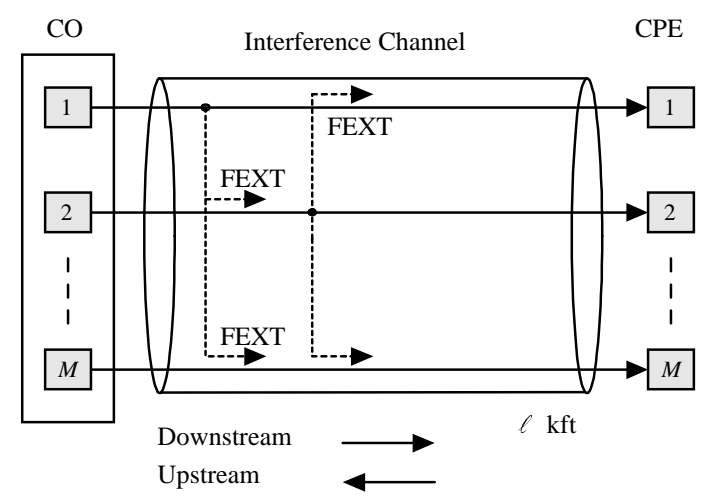

Figure 1. Downstream FEXT in $M$-ADSL lines.

\subsection{The System Model}

In this work, we are interested in downstream transmissions only and a typical system of $M$-ADSL lines is shown in Figure 1. In order to estimate the system performance as new services are activated, the bit allocation algorithm of Section 2.1 is used. The number of active sources in the bundle increases as new ADSL connections are established. The first activated line experiences

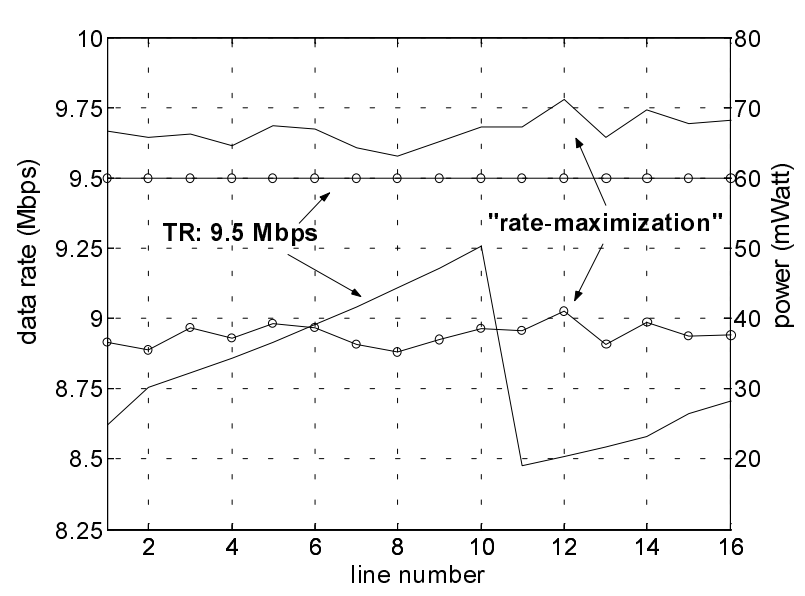

Figure 2. Power and achievable data rates in a 6-kft/16lines system under "rate-maximization" and "target-rate" (TR) strategies. The marked lines refer to data rate.

only background noise. The second line experiences background noise and FEXT from the first line. Finally the $M^{\text {th }}$ line experiences background noise and FEXT from all other lines. A modem may re-initialize if the BER increases substantially in any of its subchannels. After all $M$ lines have been initialized the margin degradation for each source is evaluated. If the system margin of any of the subchannels becomes negative, the modem re-evaluates its bit-allocation profile. This process continuous until the margin degradation for all sources becomes nonnegative and the system steady-state has been reached.

\section{3. “TARGET-RATE" ANALYSIS}

We now apply the bit-loading process described in the previous section for various $\ell$ and $M$ values. The FEXT power transfer function of several disturbers is modeled according to [2], a floor of $-140 \mathrm{dBm} / \mathrm{Hz}$ AWGN and a $40 \mathrm{kHz}$ lower band-edge are assumed. The twisted pairs are considered to be 26 AWG, no coding is applied and system margin of $6 \mathrm{~dB}$ is required.

Figure 2 compares the performance of each line in a bundle of $16-\mathrm{ADSL} / 6 \mathrm{kft}$ lines under the "rate-maximization" strategy and a "target-rate" strategy of $9.5 \mathrm{Mbps}$, when all services have been activated sequentially and the system has reached its steady-state. The "rate-maximization" strategy achieves lower data rate than the "target-rate" strategy. Actually the "rate-maximization" approach forces the modems to use the maximum allowable power in each subchannel. As a result, the noise each modem contributes to the system is maximum. In fact with only a few lines initialized in the system, the achievable data rate is higher than it is shown in Figure 2. However as more lines are activated, the margin degradation, due to maximum noise contribution from the "later" initialized modems, results in data rate degradation, since re-initialization is required in some cases. From Figure 2 it can be derived that the total data rate obtained with the "targetrate" strategy is also higher. For TR=9.5 Mbps, the binder capacity increases about $5 \%$.

Similar simulation results are obtained for other combinations of $\ell$ and $M$. Figure 3 and 4 plot the average data rate and power versus a "target-rate" constraint for a $6 \mathrm{kft}$ system respectively, 


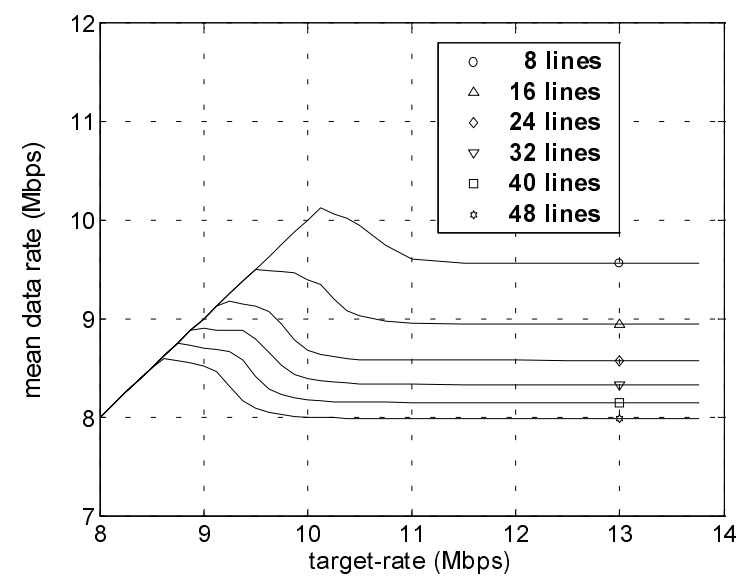

Figure 3. Rate performance of $6 \mathrm{kft}$ bundle system.

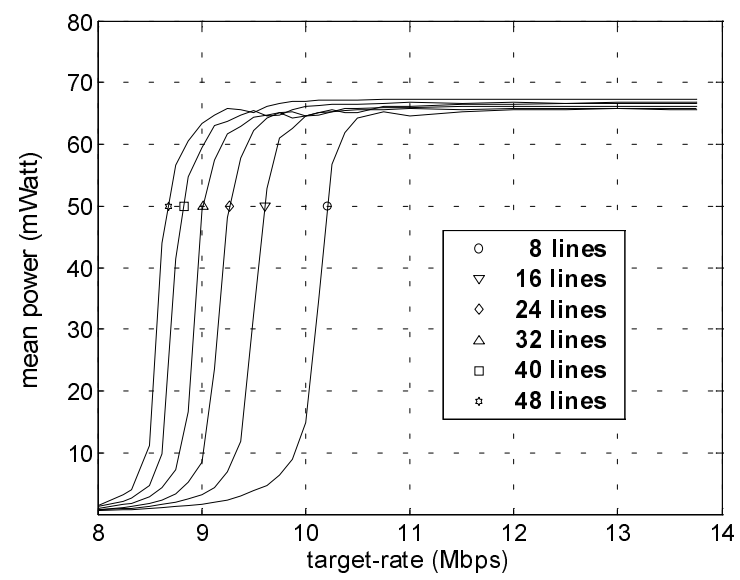

Figure 4. Power performance of $6 \mathrm{kft}$ bundle system.

while Figures 5 and 6 refer to a $10 \mathrm{kft}$ system. In the mean rate plots solid lines correspond to the "target-rate" strategy, while marks correspond to the "rate-maximization" strategy. The maximum value for the target-rate constraint of each system was selected to be the maximum rate a single user in the system may get. From these plots we can observe that all systems define a linear region of operation where all users meet the target-rate constraint. As the target-rate increases, the linearity regarding the average data rate cannot be achieved and the systems converge to the "rate-maximization" strategy's point of operation. This behavior is explained by the fact that when the system is not in the linear region of operation, some modems cannot achieve the required target-rate, even using maximum power. Power increase results in noise level increase that forces the system to reevaluate its bit-allocation and power profiles and finally to converge to a rate less than the target and equal to the point of operation of the "rate-maximization" strategy.

The optimum point of operation, in the sense of mean data rate, differs as the loop length increases. The $6 \mathrm{kft}$ system meets the optimum point at the end of its linear region, while the $10 \mathrm{kft}$ system meets its optimum performance at a target-rate that does not belong to the linear region. In fact due to the strong

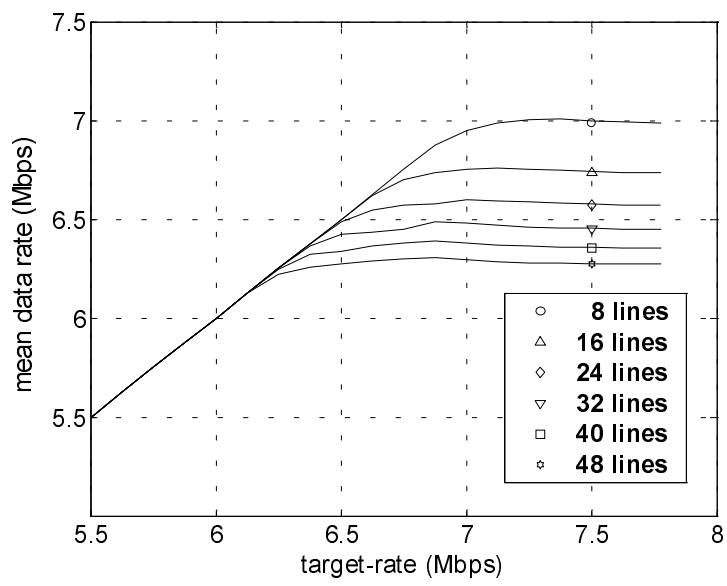

Figure 5. Rate performance of $10 \mathrm{kft}$ bundle system.

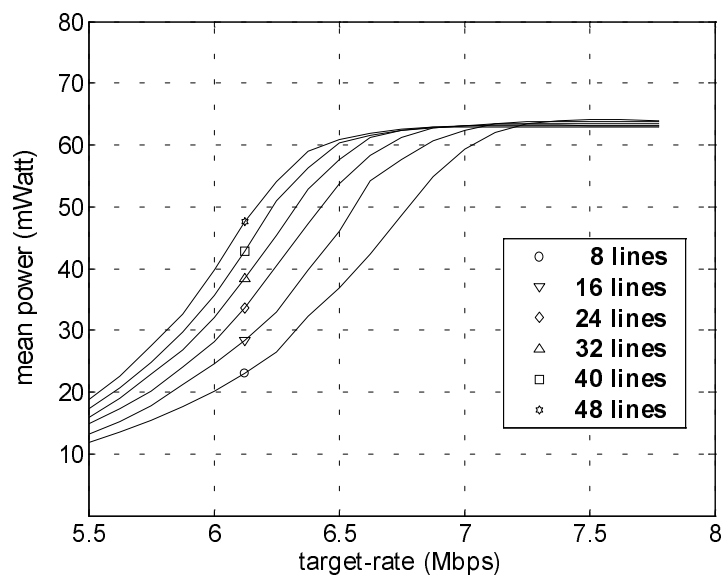

Figure 6. Power performance of $10 \mathrm{kft}$ bundle system.

attenuation of long loops over both user and crosstalk signals, the rate degradation is less sharp than in short loops, since the modems transmit using high power even for medium data rates.

Figure 7 shows the variance along with the mean data rate in a 6 $\mathrm{kft}$ system for 8 and 24 users. As it is shown, when the system does not operate in its linear region, as defined by the data rate curve, the variance increases rapidly until a maximum point and then vanishes as the system converges to the point of operation of the "rate-maximization" strategy. This operation defines a region of target-rates with high "unfair" behavior. This region corresponds to the situation where all modems transmit with high power but only a few are able to meet the rate constraint. However this failure strongly depends on the time each modem was activated relative to the total number of users in the binder, since the later-activated modems experience less noise increase originated from the initialization of new services. Therefore, the early-activated modems may execute re-initialization and may not meet the data rate constraint under the new system conditions. This behavior results to non-uniform distribution of the available bandwidth between lines with the same characteristics and belonging to the same bundle, since no information is shared between the modems located at the CO. 


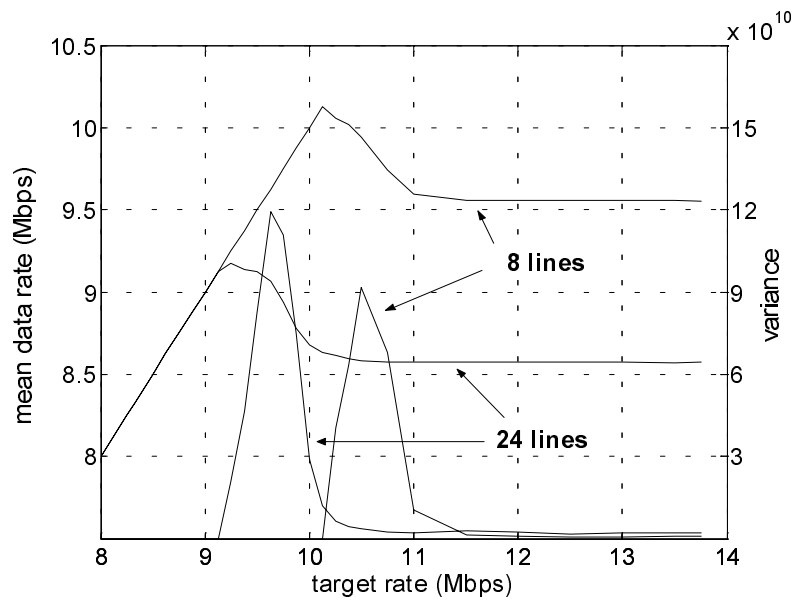

Figure 7. Mean data rates and their variance obtained by the non-cooperative single-user bit-allocation approach.

\section{IMPROVED BIT-LOADING}

This section presents a method for solving the "unfairness" of the total data rate distribution. A CO management unit can be used that collects information about the downstream links and determines the mean data rate achieved by the system. Then the CO management unit instructs all modems that achieved higher than the mean rate to decrease their data rate until they achieve the mean value, using the bit-removal algorithm under the current steady-state PSD and margin distributions. As the next step, the CO management unit forces all users that achieved lower than the mean data rate to perform rate increase using a bit-addition algorithm, where the least power expensive subchannel is filled with one more bit until the data rate or the power budget constraint is met. The bit-addition algorithm continues until the new target data rate (equal to the previously computed mean data rate) has been achieved. At this point the variance of the user data rates is minimum. The final step is to provide a minimum margin control for all margin distributions.

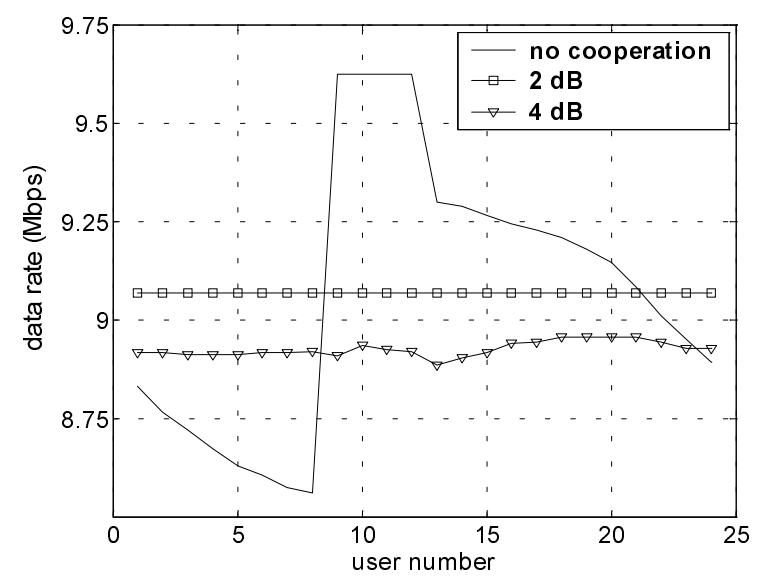

Figure 8. Data rate variance cancellation under minimum margin constraint.
During this step, all modems compute their margin distributions for all subchannels. If the margin is less than the minimum, then they scale the power of each subchannel in order to meet the margin constraint, unless the maximum transmit PSD of each subchannel is exceeded. In this case the modems remove one bit from that specific subchannel and the minimum margin control continues until all users meet the margin constraint.

Figure 8 demonstrates the effect of the additional bit-loading method for the "24-users" system of Figure 6 and for target-rate that results in maximum data rate variance. The non-marked line corresponds to the steady-state rate distribution prior to the proposed extension of the bit-loading mechanism. For up to $2 \mathrm{~dB}$ minimum margin, all users are able to achieve the average data rate. For the case of $4 \mathrm{~dB}$ margin constraint, the mean data rate decreases slightly (less than $2 \%$ ), but the "unfairness" of the noncooperative bit-allocation algorithm has been vanished.

\section{CONCLUSION}

This paper examined the performance of a system of ADSL lines belonging to the same binder. The "rate-maximization" strategy under the single-user bit-allocation scheme results to nonoptimum performance in terms of the total data rate. This singleuser approach results also in a non-uniform distribution of the achievable data rate, especially near the optimum "target-rate". In this paper we proposed an extension to this bit-loading mechanism that resolves the "unfairness" problem. This proposal is based on the cooperation between the different modems using the CO management unit. The improved bit-loading scheme achieves data rate variance cancellation and fair distribution of the total rate, under a minimum system margin requirement. This work can be extended further in order to derive a method for estimating the optimum "target-rate" in xDSL systems with different line lengths and uniform data rates.

\section{REFERENCES}

[1] Starr T., Cioffi J. M. and Silverman P. J. Understanding Digital Subscriber Line Technology, Englewood Cliffs, NJ: Prentice Hall, 1999.

[2] "Spectrum Management For Loop Transmission Systems", ANSI. ANSI-T1.417-2001.

[3] Leke A. and Cioffi J. M. "A maximum rate loading algorithm for discrete multitone modulation systems", in Proc. IEEE GLOBECOM '97, Nov. 1997, pp. 1514-1518.

[4] Sonalkar R. V. and Shively R. R. "An Efficient Bit-Loading Algorithm for DMT Applications". IEEE Communications Letters, 4(3): 80-82, 2000.

[5] Cioffi J. M. et al. "Proposed Scope and Mission for Dynamic Spectrum Management”. T1E1.4/2001-188R4, Greensboro, NC, Nov. 2001.

[6] "Asymmetrical digital subscriber line (ADSL)", ITU. ITUG.992.1, July 1999.

[7] Cioffi J. M. "A Multicarrier Primer". T1E1.4/91-159, Nov. 1991.

\footnotetext{
- This work was partially supported by the "Karatheodoris" R\&D program of the University of Patras and Project 00BE33 "Digital Subscriber Lines Technology" of the Greek Ministry of Industry.
} 